\title{
TENDÊNCIA DOS EFEITOS GENÉTICOS DIRETOS E MATERNOS DO PESO A DESMAMA DE BOVINOS DA RAÇA NELORE MOCHA NA REGIÃO PECUÁRIA CAMPO GRANDE E DOURADOS - MATO GROSSO DO SUL
}

(Tendency of the direct and maternal genetic effects on the weight at the weaning of Nelore bovines at the cattle raising region of Campo Grande and Dourados)

\author{
GARCIA, F.Q.'; FERRAZ FILHO, P.B..; SOUZA, J.C. ${ }^{3}$; SILVA, L.O.C. ${ }^{4}$ \\ ${ }^{1}$ Bolsista PIBIC/CNPq. - Departamento de Ciências Naturais - UFMS - Av,. Ranulpho Marques Leal, 3484, \\ 79603-011 - Três Lagoas - MS. pbferraz@ceul.ufms.br; \\ ${ }^{2}$ Departamento de Ciências Naturais - UFMS; \\ ${ }^{3}$ Departamento de Zootecnia - UFPR.
}

RESUMO - Dados relativos a 2891 observações de pesos de animais da raça Nelore Mocha, nascidos e criados na região pecuária Campo Grande - Dourados, no Estado de Mato Grosso do Sul, no período de 1975 a 1996, foram analisados com o objetivo de avaliar as mudanças genéticas aditivas diretas e maternas, dos pesos à desmama, padronizados para os 205 (P205) dias de idade. A tendência genética foi estimada pela regressão ponderada das médias anuais dos valores genéticos (aditivo e materno) sobre o ano de nascimento do animal. As estimativas dos componentes de (co) variância utilizadas para o cálculo dos valores genéticos, foram obtidas pelo método de máxima verossimilhança restrita livre de derivadas (REML), usando o aplicativo MTDFREML (sob modelo acima). No modelo foram incluídos os efeitos aleatórios aditivo direto e materno, além dos efeitos fixos de grupo de contemporâneos (fazenda, sexo, estação e ano de nascimento do animal) e a covariável idade da vaca ao parto (efeitos linear e quadrático). As tendências genéticas estimadas dos efeitos diretos e maternos foram $0,78 \mathrm{~kg} / \mathrm{ano}$ e $-0,35 \mathrm{~kg} / \mathrm{ano}$, respectivamente. A evolução do ganho genético oriundo do efeito aditivo direto foi de -0,51 a 2,14 kg/ano, resultando em uma amplitude de $0,1262 \mathrm{~kg} / \mathrm{anno}$, e a mesma medida da dispersão da tendência genética do efeito materno foi de $-0,0611 \mathrm{~kg} / \mathrm{ano}$, conseqüente de uma de variação de 0,27 a $-1,02 \mathrm{~kg} / \mathrm{ano}$ no período estudado. Considerando a variabilidade genética existente nos rebanhos da região, os resultados observados estão bem aquém das mudanças possíveis.

Palavras chave: gado de corte, herdabilidade, mudança genética.

ABSTRACT - Data related to 2891 observations of weights of Nelore Polled breed, borned and servants in the region of Campo Grande - Dourados, in the state of South Mato Grosso, in the period from 1975 to 1996, were analyzed with the objective of evaluating the direct and maternal addictive genetic trends. The weights were standardized for the 205 (P205) days of age. The genetic trends were dear for the pondered regression of the annual averages of the genetic values (addictive and maternal) on the year of birth of the animal. The estimates of the components of (co) variance used for the calculation of the genetic values, were obtained by the method of maximum restricted verisimilitude free from having derived (REML), using the application MTDFREML (animal models) contends direct and maternal addictive aleatory effects, besides the fixed effects of contemporaries' group (farm, sex, season and weight of the animal) and the co-variable age of the cow to the childbirth (lineal and quadratic). The esteemed genetic trends of the direct and maternal effects were $0,78 \mathrm{~kg} /$ year and $-0,35 \mathrm{~kg} / \mathrm{year}$, respectively. The evolution of the genetic gain originating from of the direct addictive effect was from $-0,51$ to $2,14 \mathrm{~kg} / \mathrm{year}$, resulting in a width of $0,1262 \mathrm{~kg} / \mathrm{year}$, and the same measure of the dispersion of the genetic trends of the maternal effect was of $-0,0611 \mathrm{~kg} / \mathrm{year}$, as a consequence from a variation of 0,27 to $-1,02 \mathrm{~kg} / \mathrm{year}$ in the period. Considering the existent genetic variability in the region, the observed results are bellow possible changes.

Key words: beef cattle, heritability, genetic trends, wheigth. 


\section{Introdução}

A ampla variabilidade de ambientes disponíveis para a pecuária brasileira levou a necessidade da subdivisão do país em regiões homogêneas de produção (ARRUDA e SUGAI, 1994). A região Campo Grande - Dourados, formada pelo agrupamento das microrregiões homogêneas de Bodoquena, Pastoril de Campo Grande e Dourados, no Mato Grosso do Sul, apresenta uma diversidade de ecossistemas que abre espaço para a criação de vários biótipos, nos seus diferentes sistemas de produção. A procura de animais adequados para diferentes ecologias, é essencial no desenvolvimento do trabalho de zoneamento ecológico da pecuária bovina. Considerandose a importância do melhoramento genético para o desenvolvimento regional, torna-se evidente a necessidade não só de implementação de programas de seleção adequados, bem como a importância de se avaliar os progressos genéticos alcançados ao longo do tempo (PIMENTA FILHO, 1986; FERRAZ FILHO et al., 1997; MELLO, 1999; SILVA et al., 1997; SOUZA et al., 1998 e FERRAZ FILHO et al., 2002).

A seleção genética constitui-se a mais importante ferramenta do melhoramento animal disponível aos melhoristas e criadores que desejam promover aumento da eficiência de produção de bovino de corte no país. A escolha dos indivíduos com base em seus méritos genéticos tem se tornado a forma mais comum e certamente, a mais eficiente de se promover seleção. A despeito disso, os progressos genéticos serão concretizados se, além de se observar à escolha de animais que serão progenitores da próxima geração, for assegurado que eles sejam realmente utilizados com maior intensidade sendo, portanto, progenitores de maior número de filhos na geração seguinte. Assim, torna-se importante proceder-se o acompanhamento dos progressos genéticos acumulados ao longo do tempo em projetos de seleção, não só como forma de se quantificar a mudança genética que vem se processando, mas também, e principalmente, no sentido de se avaliarem os benefícios e se procederem os ajustes quando necessários (EUCLIDES FILHO et al., 1997b).
Com o intuito de se ter avaliação da mudança genética na população controlada da raça Nelore Mocha na região pecuária Campo Grande - Dourados, no Mato Grosso do Sul, serão feitas avaliações das tendências dos efeitos genéticos direto e maternos pela regressão dos valores genéticos médios anuais sobre o ano de nascimento dos animais.

\section{Material e Métodos}

Os dados usados neste estudo foram extraídos do arquivo do Controle de Desenvolvimento Ponderal da Associação Brasileira de Criadores de Zebu (ABCZ). As informações referem-se a pesagens a desmama, padronizadas para os 205 dias (P205), de 2749 animais da raça nelore Mocha, nascidos no período de 1975 a 1996 e criados em regime de pasto em 49 fazendas localizadas na região pecuária Campo Grande - Dourados. As estimativas dos componentes de (co)variância e dos parâmetros genéticos foram obtidas pelo programa MTDFREML (BOLDMAN et al., 1995), que utiliza a metodologia da máxima verossimilhança restrita livre de derivadas (DTREML). Foi adotado o modelo animal que incluiu os efeitos fixos de grupo contemporâneo (fazenda, sexo, estação e ano de nascimento do animal), idade da vaca como covariável (linear e quadrática) e os efeitos aleatórios genéticos diretos e maternos. Desta foi utilizado o seguinte modelo:

$$
Y=X \beta+Z a+M m+e
$$

em que $Y$ é o vetor da variável dependente (P205); $\beta$, o vetor de efeitos fixos no modelo, associados com a matriz de incidência $X ; a$, o vetor dos efeitos aleatórios de valor genético aditivo direto do animal, associado com a matriz de incidência $Z ; m$, o vetor dos efeitos genéticos maternos, associado com a matriz de incidência $M$; e e; o vetor dos efeitos residuais aleatórios.

Estes componentes foram utilizados para predizer os valores genéticos para efeito genético direto e materno dos pesos aos 205 de idade. As tendências genéticas (aditiva e materna) foram estimadas por análise de regressão linear, das médias ponderadas dos valores genéticos, em função do ano de nascimento do animal, utilizando o programa SAS (1996). 
Tendência dos efeitos genéticos diretos e maternos do peso a desmama de bovinos da raça nelore mocha...

\section{Resultados e Discussão}

A média e o respectivo desvio padrão foram $168,05(27,60) \mathrm{kg}$, com coeficiente de variação de $16,43 \%$. Esta média que representa $35 \%$ do peso mínimo considerado para abate $(480 \mathrm{~kg})$, pode ser considerada relativamente baixa, visto que animais com baixos pesos nas idades iniciais do desenvolvimento demandam um período maior de recria, elevando, conseqüentemente, os custos na propriedade.

As estimativas dos componentes de variância e covariância, bem como das herdabilidades e da correlação entre os efeitos diretos e maternos, são apresentadas na TABELA 1. A estimativa de herdabilidade aditiva direta para P205 apresentou valor médio $(0,54)$, tal estimativa indica que os trabalhos de seleção tenham uma perspectiva razoável de êxito.Também foi significante o valor da estimativa de herdabilidade dos efeitos aditivos maternos $(0,27)$ indicando que os mesmos exercem importante influência sobre o peso a desmama.

\section{TABELA 1 - COMPONENTES ${ }^{1}$ DE VARIÂNCIAE COVARIÂNCIA, HERDABILIDADES DIRETAE MATERNA ${ }^{1} E$ CORRELAÇÃO GENÉTICA ENTRE OS EFEITOS DIRETOS E MATERNO ${ }^{1}$ PARA PESOS AOS 205 (P205) DIAS DE IDADE, EM BOVINOS DA RAÇA NELORE. (n=2891). MATO GROSSO DO SUL.

\begin{tabular}{ccccccccc}
\hline Peso & $\hat{\sigma}_{P}^{2}$ & $\hat{\sigma}_{a}^{2}$ & $\hat{\sigma}_{m}^{2}$ & $\hat{\sigma}_{e}^{2}$ & $\hat{\sigma}_{a m}$ & $\hat{h}_{a}^{2}$ & $\hat{h}_{m}^{2}$ & $\hat{r}_{G w m}$ \\
\hline P205 & 530,45 & 286,43 & 143,16 & 248,08 & $-147,22$ & $0,54(0,09)$ & $0,27(0,13)$ & $-0,73$
\end{tabular} \\ ${ }^{1} \hat{\sigma}_{p}^{2}, \hat{\sigma}_{a}^{2}, \hat{\sigma}_{m}^{2}, \hat{\sigma}_{e}^{2}, \hat{\sigma}_{a m} \hat{h}_{a}^{2} \hat{h}_{m}^{2}, \hat{r}_{G m}=$ componentes de variância fenotípica, genética aditiva direta, genética aditiva matema, e residual e de covariância genética entre os efeitos aditivos direto e materno, herdabilidade direta e matema, e correlação genética entre os efeitos diretos e maternos, respectivamente.}

O valor da correlação genética entre os efeitos genético direto e materno foi negativo $(-0,73)$, demonstrando certo antagonismo entre estes efeitos, indicando que métodos de seleção que consideram estes dois efeitos resultariam em maior resposta econômica à seleção em longo prazo do que a seleção baseada unicamente no efeito genético direto (ELER et al. 1996).

FIGURA 1 - TENDÊNCIA GENÉTICA (KG) DO EFEITO GENÉTICO ADITIVO DIRETO, PARA O PESO AO DESMAME, PADRONIZADO PARA OS 205 DIAS (P205), NO PERÍODO DE 1975 A 1996, EM BOVINOS DA RAÇA NELORE. MATO GROSSO DO SUL. $(n=2891)$.
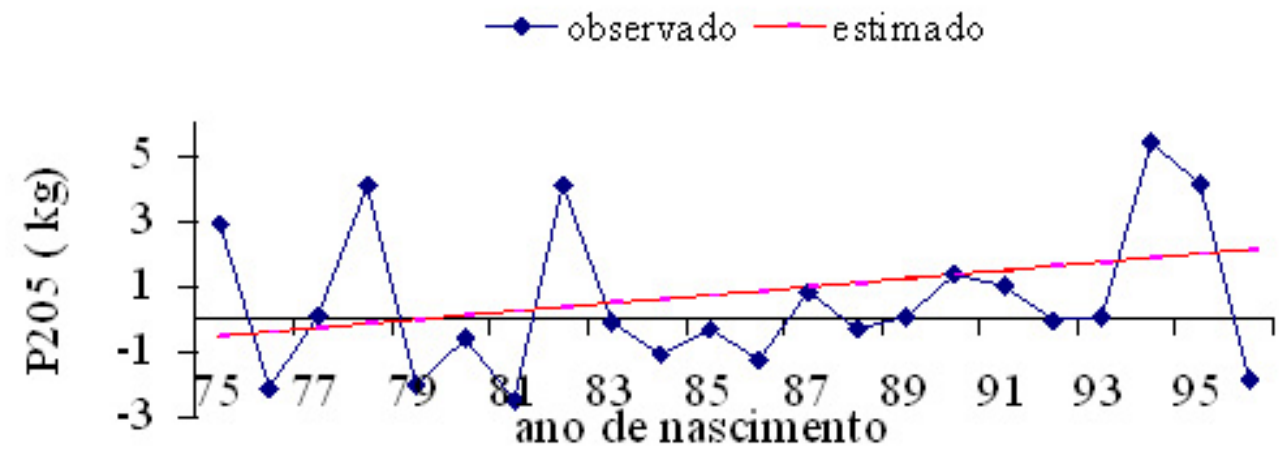
A média estimada do valor genético direto foi de $0,78 \mathrm{~kg} / \mathrm{ano}$, representando, em termos de mudança genética anual incrementos de $0,46 \%$. Na FIGURA 1 é apresentada a variação dos valores genéticos observados e estimados do efeito aditivo direto para pêso à desmama, ao longo dos anos, onde pode-se constatar variação do ganho genético de -0,51 a 2,14 kg/ ano, resultando em amplitude de 0,1262 kg/ano. Resultados indicando baixos progressos genéticos, não são raros, como os obtidos em diversos rebanhos de diferentes raças, por PACKER (1977), ELER et al. (1994), EUCLIDES FILHO et al. (1997a,b), SILVA et al. (1997), FERRAZ FILHO et al. (2002) e SOUZA et al. (1998).

A tendência genética para o efeito materno apresentou-se negativa $(-0,35 \mathrm{~kg} / \mathrm{ano})$ possivelmente em função da correlação negativa com o efeito genético aditivo direto. Este valor foi significativo $(P<0,001)$, o que demonstra estar ocorrendo declínio anual na porção materna, correspondendo a perdas anuais de $0,21 \%$ em relação à média para os pesos, como observado na FIGURA 2. Estes valores obtidos, que regridem o ganho genético no decorrer dos anos, reforçam a citação de BRADFORD (1972) sobre a necessidade de programas designados a melhorar o desempenho materno, não apenas por causa da contribuição deste componente, mas também pelo aparente do efeito materno na expressão da variação genética na taxa de crescimento.

FIGURA 2 - TENDÊNCIA DOS EFEITOS GENÉTICOS MATERNOS PARA PESO ADESMAMA, PADRONIZADO PARA 205 DIAS (P205), NO PERÍODO DE 1975 A 1995, EM BOVINOS DA RAÇA NELORE. MATO GROSSO DO SUL. $(n=2891)$.
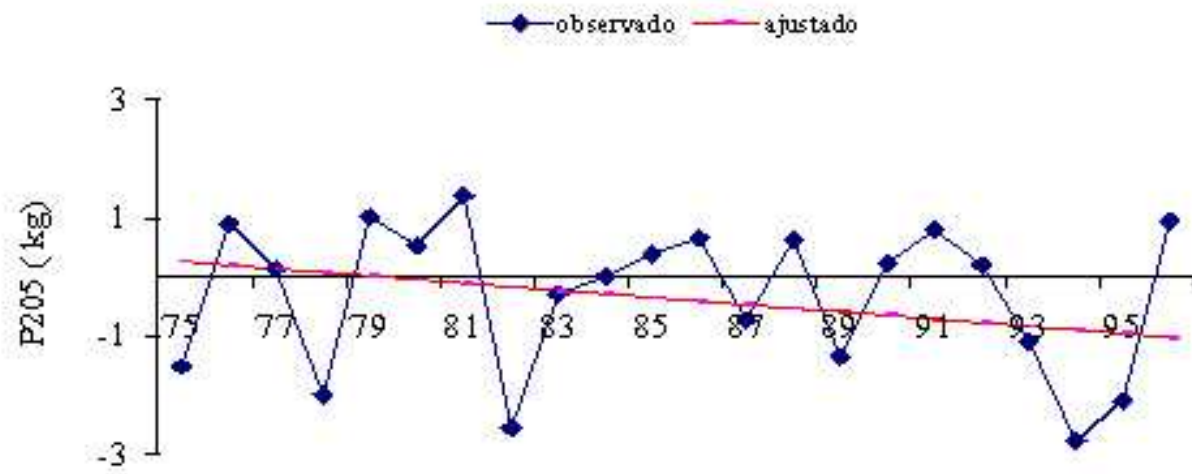

ano de nascimento

Considerando-se o período médio de sete anos como o intervalo de gerações em bovinos da raça Nelore Mocha (FARIA et al., 2001), a intensidade de seleção (i) de 1,274 (retenção de $10 \%$ de machos e $50 \%$ de fêmeas), a herdabilidade direta ( ) da TABELA 1, o desviopadrão fenotípico $\left(\sigma_{p}\right)$ igual a $23,03 \mathrm{~kg}$, seria possível obter avanços genéticos $(\Delta \mathrm{G})$ por geração $\left(\Delta \mathrm{G} /\right.$ geração $\left.=\mathrm{i} \times \hat{h}_{a}^{2} \times \sigma_{\mathrm{p}}\right)$ da ordem de $2,26 \mathrm{~kg} / \mathrm{ano}$, para P205, correspondendo a $1,35 \%$ da médias dos pesos. Utilizando-se a herdabilidade total $\left.\hat{h}_{T}^{2}=\mid \hat{\sigma}^{2}+0,5 \hat{\sigma}^{2}+1,5 \hat{\sigma}_{a x} / \hat{\sigma}^{2}\right]$, e tomando-se os resultados da TABELA 1 , os ganhos possíveis seriam menores em virtude do antagonismo entre os efeitos aditivos direto e materno e exatamente igual a média observada de 0,78 kg/ano, correspondendo, conforme citado, a
$0,46 \%$ da média dos pesos dos rebanhos. Segundo SMITH (1985), a taxa de mudança genética anual possível de se obter é de 1 a $3 \%$ da média da população. Desse modo observa-se que, as mudanças genéticas anuais situam-se abaixo do limite inferior, daquele intervalo quando são apreciados os efeitos maternos, e com a redução do intervalo de gerações, os ganhos genéticos aproximam-se daquela demarcação.

\section{Conclusões}

Os pesos aos 205 dias têm suficiente herdabilidade para permitir rápidas mudanças através da seleção. O antagonismo genético observado entre os efeitos genéticos diretos e maternos indica que a seleção intensiva para 
Tendência dos efeitos genéticos diretos e maternos do peso a desmama de bovinos da raça nelore mocha...

crescimento individual pode resultar em redução na performance materna, sugerindo que este componente não seja negligenciado em programas de melhoramento.

As tendências genéticas aditivas observadas evidenciam a necessidade de se utilizar genótipos superiores nos rebanhos, devendose porém, estar atento à variabilidade do rebanho, buscando-se continuadamente utilizar os melhores reprodutores.

\section{Referências}

ARRUDA, Z.J.; SUGAI Y. Regionalização da pecuária bovina no Brasil. Campo Grande: EMBRAPA-CNPGC; Brasília: EMBRAPA-SPI, 1994. 144p - (EMBRAPA-CNPGC, documentos, 58).

BOLDMAN, K.G.; KRIESE, L.A.; VAN VLECK, L.D. A Manual for Use of MTDFREML; a Set of Programs to Obtain Estimates of Variances and Covariances [DRAFT]. Lincoln, Department of Agriculture, Agricultural Research Service, 1995. 120p.

BRADFORD, G.E. The role of maternal effects in animal breeding: VII. Maternal effects in sheep. Journal of Animal Science, v.35, n.6, p.1324-1334, 1972.

ELER, J.P.; FERRAZ, J.B.S.; LÔBO, R.B. Estimação da tendência genética na raça Nelore, usando modelos animais univariado e multivariado. In: REUNIÃO ANUAL DA SOCIEDADE BRASILEIRA DE ZOOTECNIA, 31, 1994. Maringá. Anais... Maringá: Sociedade Brasileira de Zootecnia, 1994. p.171.

ELER, J.P.; FERRAZ, J.B.S.; SILVA, P.R. Estimação simultânea de parâmetros genéticos para características de importância econômica na raça Nelore, com a utilização de modelos animais. In: REUNIÃO ANUAL DA SOCIEDADE BRASILEIRADE ZOOTECNIA, 33, 1996. Fortaleza. Anais... Fortaleza: Sociedade Brasileira de Zootecnia, 1996. p.99-101.

EUCLIDES FILHO, K.; SILVA, L.O.C.; ALVES, R.G.O.; NOBRE, P.R.C. Tendências genéticas na raça Indubrasil. In: REUNIÃO ANUAL DA SOCIEDADE BRASILEIRA DE ZOOTECNIA, 34, 1997. Juiz de Fora - MG. Anais... Juiz de Fora: SBZ, 1997a. p.171.

EUCLIDES FILHO, K.; SILVA, L.0.C.; FIGUEIREDO, G.R. Tendências genéticas na raça Guzerá. In: REUNIÃO ANUAL DA SOCIEDADE BRASILEIRA DE ZOOTECNIA, 34, 1997. Juiz de Fora - MG. Anais... Juiz de Fora: SBZ, 1997b. p.173.
FARIA, F.J.C.; VERCESI FILHO, A.E.; MADALENA, F.E.; JOSAKIAN, L.A. Intervalo de gerações e tamanho efetivo da população na raça Nelore Mocho In: REUNIÃO ANUAL DA SOCIEDADE BRASILEIRA DE ZOOTECNIA, 38, 2001. Piracicaba - SP. Anais... Piracicaba: SBZ, 2001 p.483-484.

FERRAZ FILHO, P.B.; BIANCHINI SOBRINHO, E.; SILVA, L.O.C.;ALENCAR, M.M.; SOUZA, J.C. Tendência genética em pesos de bovinos da raça Nelore Mocha no Brasil. In: REUNIÃO ANUAL DA SOCIEDADE BRASILEIRA DE ZOOTECNIA, 34, 1997. Juiz de Fora MG. Anais... Juiz de Fora p.254-256, 1997.

FERRAZ FILHO, P.B.; RAMOS, A.A.; SILVA, L.O.C.; SOUZA, J.C.; ALENCAR, M.M.; MALHADO, C.H.M. Tendência genética dos efeitos direto e materno sobre os pesos à desmama e pós-desmama de bovinos da raça Tabapuã no Brasil. Revista Brasileira de Zootecnia, Viçosa, v.31, n.2, p.635-640, 2002.

MELLO, S.P. Tendência genética para pesos em um rebanho da raça Canchin: Jaboticabal, SP: FCAV, 1999. 78p. Dissertação (Mestrado em Zootecnia) - Faculdade de Ciências Agrária e Veterinárias. Campus de Jaboticabal/Universidade Estadual Paulista, 1999.

PACKER, I.U. Análise genética do crescimento até a desmama de bezerros Canchin. Piracicaba, SP, USP, 1977. 173p. Tese (livre Docência). Escola Superior de Agricultura "Luiz de Queiroz", Universidade de São Paulo, 1977.

PIMENTA FILHO, E.C. Mudança genética no peso aos 365 dias de idade de bovinos Nelore no Estado de São Paulo. Ribeirão Preto, 1986, 61p. Tese (Doutorado em Genética) - Faculdade de Medicina de Ribeirão Preto, Universidade de São Paulo.

SILVA, L.O.C.; EUCLIDES FILHO, K.; NOBRE, P.R.C.; JOSAHKIAN, L.A. Tendência genética na raça Nelore no Brasil. In: REUNIÃO ANUAL DA SOCIEDADE BRASILEIRA DE ZOOTECNIA, 34, Juiz de Fora, 1997. Anais... Juiz de Fora : Sociedade Brasileira de Zootecnia. 1997, p. 175.

SMITH, C. Rates of genetic change in farm livestock. Research Develop. Agric., v.1, n.2, p.79-85, apud Animal Breeding Abstracts, v.53, n.9, p. 699, 1985. SOUZA, J.C.; RAMOS, A.A.; SILVA, L.O.C.; EUCLIDES FILHO, K.; ALENCAR, M.M.; FERRAZ FILHO, P.B. Tendência genética do peso ao desmame de bezerros da raça Nelore In: REUNIÃO ANUAL DA SOCIEDADE BRASILEIRA DE ZOOTECNIA, 35, Botucatu, 1998. Anais... Juiz de Fora: Sociedade Brasileira de Zootecnia. 1998, p. 231. 